\title{
Project Space of the Region: Content, Structure, Integration Imbalances
}

\author{
Mira Kantemirova ${ }^{1, *}$, Alla Gurina $^{1}$, Marina Miroshnichenko ${ }^{2}$, Z.L. Dzakoev ${ }^{3}$, and Lyudmila \\ Olisaeva $^{4}$ \\ ${ }^{1}$ North Ossetian state medical Academy, 362025 Vladikavkaz, Russia \\ ${ }^{2}$ Kuban state technological University, 350072 Krasnodar, Russia \\ ${ }^{3}$ North Ossetian state University, 362025 Vladikavkaz, Russia \\ ${ }^{4}$ North Caucasian Mining and Metallurgical Institute (State Technological University) of the Republic \\ of North Ossetia-Alania, Vladikavkaz, Russia
}

\begin{abstract}
The results of the study of the project space of the region have been presented. The purpose of the study is to determine the content and structure of the region's project space, the reasons for the emergence of integration imbalances therein, based on an overview of the state of the problem in scientific sources and an empirical analysis in the region.

The following research methods have been used: the method of comparative analysis of specific projects of various types and documents representing them (summaries, abstracts, etc.); the method of correlation analysis. These methods have been modified to identify the links and dependencies between the projects, the mutual influence of their characteristics. The sample consisted of 1200 projects. For comparison purposes, the samples of projects and programs from different regions of Russia have been used.

In the course of the study, the following results have been obtained: 1 . The prerequisites for the study of the design space of the region have been determined, its content has been revealed. 2 . The analysis of the project space of the region has been carried out, which made it possible to determine the structure of the projects, the peculiarities of the correlation between them, the content of integration imbalances, the reasons for their formation and the main types thereof. 3. The main approaches to the development of the project space of the region, reduction of the negative impact of integration imbalances therein have been presented. The research of the project space of the region has not been conducted before, it is unique and new. At the same time, they can be useful in the formation of a project society, the methodology of which is being developed in many countries of the world.
\end{abstract}

\section{Introduction}

It was not possible to find any references directly on the content of the project space (company, region, state). The relevance of the topic is due to the fact that the ontological interest in the study of the project space is caused by the widespread use of the project

\footnotetext{
* Corresponding author: kantemirova.mira@mail.ru
} 
approach in business, non-profit organizations, municipal, regional and state administration. A huge number of various projects are carried out by the individuals, companies, corporations, regions, countries and their associations in order to meet their needs and interests, acting as centers for the formation in their environment of specific forms of organization and interaction that require research and analysis.

The foundations of project management methodology took shape in the 20th century in the form of a specific discipline and practical actions applicable in various areas of human activity. Projects are understood as temporary organizations designed to obtain unique results (products, services) according to the customer's requirements and the limited time and resources. To date, project management is an independent discipline, theoretical foundations, international and national standards, regulatory documents governing its application have been developed. The objects of this discipline are the individual projects, as well as the portfolios as a set of the projects, programs and sub-portfolios, combined to achieve the strategic goals and improve an efficiency.

As a rule, a single project management is carried out by the customer (individual, management company, etc.), who independently determines the goal, methods of implementation and communication with objects of the external environment (including with other projects) based on his own needs and interests. In project management, one can distinguish a group of general management methods related to planning, personnel, performance assessment, etc., as well as specific methods: critical path analysis (CPM), Gantt chart, project analysis and evaluation (PERT) and many others.

The portfolio components can include both dependent and independent projects, but focused on a common result. Portfolio management is centralized for the set of the projects included therein and comprises of the methods of identification, categorization, and evaluation, selection, prioritization, balancing, authorization, control, etc. to achieve the strategic goals of the customer.

The relevance and prerequisites for the study of the project space of the region and the integration imbalances therein are due to the following circumstances.

First, the active development of the project approach has led to the creation of many project management methodologies, including the following: Project Management Body Of Knowledge (PMBOK), Waterfall, AGILE, PRINCE2, CCPM, Lean, XPM, Scrum, etc. [1; 2]. To support the ever-expanding range of the approaches, the Project Management Institute (PMI, USA) offers the transitional options between the traditional and adaptive methodologies, allowing for the development and implementation of the most modern projects [3]. Improving management methodologies allows you to increase the validity of indicators, the achievability of goals and the effectiveness of the implementation of each individual project [4]. However, they are difficult to use to solve complex problems at the level of interproject interaction, which are common in real practice. So, for example, using the methods discussed above, it is possible to maximize the performance of a vegetable growing project, but if it is not synchronized with other relevant projects for the production of the fertilizers, storage facilities, road construction, transportation, etc., then its real return for the region will not be as expected. In this regard, there is a need for methodological development of the project space, allowing to analyze and optimize the interaction of the projects from the standpoint of a comprehensive and promising solution to the socioeconomic problems of the region.

Second, in the regions of Russia, the institutional environment for project implementation is largely formed for their individual servicing (development, attraction of loans, etc.), which largely corresponds to the redistribution model of the economy, taking into account the interests of VIPs as part of the stakeholders. This ensures the implementation of such individual projects, but does not contribute to the creation of regional management systems based on the project approach. The institutional environment 
is the basis for the economy (production, exchange and distribution), however, it does not functionally replace the project space, which is its subsystem $[5, \mathrm{p} .6]$.

Third, the orientation of regions towards the preferential use of the projects in solving the development problems leads to the phenomenon of fragmentation of their socioeconomic space. It forms a multitude of separate areas of activity and their aggregates (zones) that are not covered by the projects, and because of this, are limited in resource provision, support, etc., which negatively affects the efficiency and synergy of socioeconomic development of the entire region. In addition, the relatively short period of the project's life contributes to the further allocation of limited resources for other unrelated projects, enhancing the differentiation of the socio-economic development of the region. Development of the theoretical foundations of the design space, its practical monitoring and condition control will reduce the negative impact of problems of this kind.

Within the borders of the region, a huge number of various projects are being implemented that determine the gross regional product, the standard of living of the population, and affect the state of society. Most of these projects are independent and carried out on their own, are not part of portfolios, are at different stages of the life cycle and are not aligned with other projects in terms of goals, resources and other indicators. From the point of view of the aggregate interests of the entire region, this situation leads to imbalances caused by the problems of integration and interaction of projects with each other and with infrastructure facilities: suboptimal distribution of tasks, work and efforts, resource overruns, missed deadlines, deterioration of performance, and decrease in project efficiency. In connection with the wide diffusion of the project approach in all spheres of society, the need to move from studying individual projects to finding a methodology for ensuring the project development of regions and entire societies is urgent, which implies the study of the essence of the project space.

The scientific hypothesis is associated with the assumption that the methodological aspects of the project approach should be supplemented with the concept of the project space, which reveals the features and contributes to the harmonization of the interaction of projects, increasing the efficiency and synergy of the social and economic development of the region.

The purpose of this article is to study the content and structure of the region's project space, the reasons for the emergence of integration imbalances therein.

Research objectives:

1. study of the theoretical background of the project space of the region;

2. conducting an empirical study of the project space in the region;

3. substantiation of the content and structure of the region's project space, the reasons for the formation of integration imbalances therein.

The subject of the article is the design space, as one of the components of the economic space, which has a certain impact on its state and the nature of development.

The object of the research is the topical issues of dynamics and phenomena currently being observed in the southern part of Russia, in particular, in the Republic of North Ossetia-Alania, which has its own space, which has features of a geographical, economic, social and other nature. While reporting in a certain conditionality and still insufficient elaboration of the concept of "project space", we, at the same time, believe that its research is quite justified and productive, because it allows not only to theoretically substantiate and reveal the attributive content of the projects being implemented, but also to identify and link the peculiarities and patterns of their integration with each other and with objects of the external environment in a single process of shaping the future appearance of the region.

The analysis of the references made it possible to note the still insufficient attention of scientists and experts to the study of the aspects of the project space, which is in clear dissonance with the active development of substantiating the project society. 


\section{Review of literature}

It was not possible to find any references directly on the content of the project space (company, region, state). The interaction of projects with each other, as well as projects at different stages of the life cycle, led to the study of the problems of transition by stages of the project, the definition of time boundaries, the temporality of these stages and transitions. Projects are considered as developing temporary organizations with several contexts $[6 ; 7 ; 8]$ and based on the project approach [9]. Completion of projects leads to the need for a transition to permanent forms of organization using the operational type of functioning [10]. In this case, the main attention of the researchers is paid to the peculiarities of the transition from the project itself to its operation [11]. It should be noted that in such a setting, the transition means a change in "cooperation between different forms of organization with different temporality" [12]. However, from the perspective of a spatial approach, projects not only change their form and relationships with other organizations, but their spatial distribution as well. At the implementation stage, the concept takes the form of a project and is classified as a project. The structures of its environment cooperate with it according to the "organization-project" scheme, which determines the presence of certain features (high risks, uncertainty of the final result, etc.). At the same time, the design concept itself, as well as the structures collaborating with it, are the elements of the project space.

In the case of transformation of the project concept in the process of transition to the organization of the operational business, its relations with the partners, accordingly, take the form of an "organization-to-organization" model. This means that the project as a concept and a temporary structure stops its life cycle, ceases to be classified as a project and is modified into a new form - an established organization that carries out the operational activities [13]. In our opinion, at the same time, such a realized project leaves the project space (if it is not further connected with other projects) together with the structures that were in business relations with it.

A similar situation arises when analyzing the implementation of interdepartmental projects [14], presented as projects of different departments, as well as large megaprojects $[15 ; 16]$, which cover many participants, departments and industries. Other projects are characterized by time, resource, organizational boundaries, as well as boundaries of change, scale and temporality. The authors consider them as spatial structures, however, they are not connected with the aspects of spatial space.

In the process of transition of a project to another state, it becomes necessary to study not only the changes in purely phase modifications and instrumental changes (organization, management, etc.), but also a number of other phenomena, for example, social and symbolic aspects of transformations $[17 ; 18]$, which, along with the project space, are also insufficiently disclosed in published sources.

When considering the projects in the form of trade zones [19], global projects [20], research of the content of the project society [21], the attention of the authors also bypassed the spatial aspects of their structure.

A significant number of studies recognize the need to link the project and the corresponding environment of its existence, which is represented by the institutional environment, organizational or infrastructural environment [22], the environment for carrying out the operational activities, etc. Environment and space are inextricably linked: the environment fills the space and determines the conditions for the implementation of projects therein.

In the research papers, a particular attention on project management is paid to the issues of "project failures", the emergence of problems during their implementation [23], which 
requires the use of special methods, for example, the use of integration between projects [24], coordination of actions [25], the use of a strategic approach [26], etc.

\section{Methods}

A set of methods was has been used to reveal the nature of the interaction of regional projects with each other and with the infrastructure entities. The analysis of the subject area of the content of specific projects has been carried out in terms of identifying their integration with each other and the emergence of synergistic links. A comprehensive analysis of a sample of projects has been carried out at the stage of their development (initiation). When identifying the projects related to each other in the subject area, further monitoring at the stage of their implementation and analysis of the results have been carried out. In addition, the correlation of the indicators in two groups of projects has been determined: non-conjugate and conjugate ones.

The empirical base of the study is represented by the projects and documents characterizing them, the results of the interviews with the participants in project activities, statistical data on the region of Russia - the Republic of North Ossetia-Alania. The study has been carried out in the period of 1996-2020 with a total coverage of 1200 projects implemented in the region, accepted for support in the corresponding years in regional documents in the form of investment projects [27], innovative projects [28] presented in the catalogs (for example: [29]), projects submitted to commercial banks, investment funds, microfinance organizations, etc. The sample of projects is representative, corresponds to the problem under study, the studied periods of time, the priorities of the activity, covers the studied aspects of the phenomenon. The structure of the projects studied by the industry, forming the project space of the region, is shown in Table 1.

Table 1. The structure of the projects under study by industry

\begin{tabular}{|l|l|l|l|}
\hline \multirow{2}{*}{$\begin{array}{l}\text { Project sectoral } \\
\text { affiliation }\end{array}$} & \multirow{2}{*}{ Share, \% } & \multicolumn{2}{|c|}{ Including owned projects, \%: } \\
\cline { 3 - 4 } & & $\begin{array}{c}\text { legal } \\
\text { entities }\end{array}$ & natural persons \\
\hline Agriculture & 12 & 4 & 8 \\
\hline Industry & 9 & 4 & 5 \\
\hline Trade & 17 & 6 & 11 \\
\hline Construction & 13 & 5 & 8 \\
\hline Transport & 6 & 3 & 3 \\
\hline Education & 3 & 2 & 1 \\
\hline Healthcare & 5 & 2 & 3 \\
\hline Services & 19 & 6 & 13 \\
\hline Other & 16 & 5 & 11 \\
\hline
\end{tabular}

The ongoing projects by industry and areas of activity reflect the basic needs and interests of the customers and participants. The study identified the relationship between the characteristics of the projects. In particular, the correlation between the budget and project duration is shown in Figure 1. 


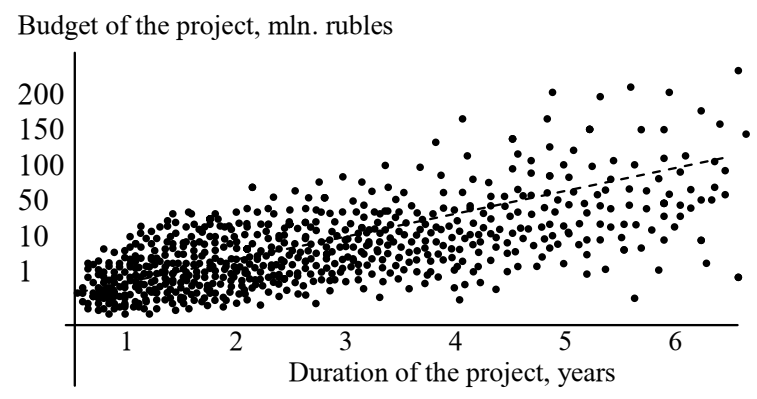

Fig. 1. Correlation scatter diagram of regional projects by budget and duration of their implementation

It follows from the diagram that most of the projects are low-budget (up to 1 million rubles) and have the terms of implementation up to 3 years. The projects with a large volume budget have longer implementation terms.

The study determined the presence of a business connection between several projects at their input and output in the form of supplies of materials, products (works, services) that contribute to an integrated focus on solving any economic problem in the region. For example, the problem of transport services to the population of the city only along one of the routes requires not only the development and implementation of the main project to create a carrier company, but also many other projects of different directions, connected with it functionally: for the repair and arrangement of the highways; construction of stops for passengers; car service; on the organization of dispatch communication; for personnel training, etc. At the same time, the involvement of the infrastructure stakeholders representing the interests of road safety authorities, environmentalists, etc., is also required to solve this problem. The absence of at least one of the projects in this interconnected system sharply worsens the economic indicators of the main project as a whole. In addition, such an integrated approach to solving problems based on a system of projects makes it possible to increase the efficiency of socio-economic indicators of the region. However, the analysis showed that only about $2 \%$ of the projects have cooperation and other links between themselves, ensuring the complexity of the project approach.

Particular attention was paid to identifying and analyzing the most representative integration imbalances that determine the nature of the interaction of the projects with each other and with the infrastructure elements. The identified integration imbalances have been revealed, ranked and systematized according to the hierarchy and the significance of their impact on the socio-economic situation in the region.

The impact of the projects on the indicators of the socio-economic situation of the region has been assessed.

\section{Results}

The basic concepts used in the research have been formulated.

The project space of the region is understood as a set of the projects, actors and stakeholders, as well as the connections, relationships and interactions between them to meet the needs and achieve the goals of socio-economic development based on the project approach.

The project management actor is the main actor implementing the project. Represents a legal or natural person, institution, individual or a group.

Stakeholders are the parties interested in and participating in the implementation of the project. 
Imbalance is understood as the absence (violation) of balance, equilibrium between the elements of the project space.

Project space properties:

The main property of the project space is associated with integration, which is understood as the unification of parts into a whole on the basis of mutual agreement. Taking into account the context of the research topic, the integration of the projects means the processes that contribute to an increase in the degree of their interconnections, interactions and interdependencies, leading to the formation of a holistic and organized project space.

- capacity - the ability to accommodate (contain) a certain number of projects and stakeholders in the space under study (overflow or absence of the elements is undesirable);

- limitation - the presence of boundaries that localize the project space;

- relativity - the project space is considered relative to other spaces, objects and phenomena of the surrounding world;

- $\quad$ specificity - the difference from other types of spaces: physical, economic, etc.;

- conjugation of the projects - the relationship of projects with each other and their mutual influence on each other.

The conjugation of the projects is considered as one of the forms of integration. When forming the project space, 2 types of the following conjugation are used. First, forward conjugation, in which the products (services, works) of a given project are consumed by a subsequent project. In this regard, both projects are in mutual influence along the added value chain. For example, a project to create a company can be paired with a subsequent project to stimulate the personnel therein, which will improve the results of the company's functioning. This, in turn, may further lead to an increase in incentive payments to company employees.

Second, backward conjugation - when a given project is linked (has a connection) with a previous project through the consumption of its products (works, services). This is a link along the value added chain of a project. For example, it may be desirable for a paperclip project to be linked to a previous metal wire project.

On the basis of integration and conjugation, the project space can be as follows:

- fragmentary (non-integral), consisting of disordered, unrelated, or insufficiently integrated elements;

- integrated - holistic, formed from ordered ones. interconnected, interacting and interdependent elements.

On the basis of belonging, it is proposed to distinguish between the project space of an individual implementing a personal project, a company, a region and a state.

The nuclei of the project space formation are the projects that concentrate the resources, infrastructure, and communications around them.

The composition of the project space in terms of one project is shown in Figure 2. 


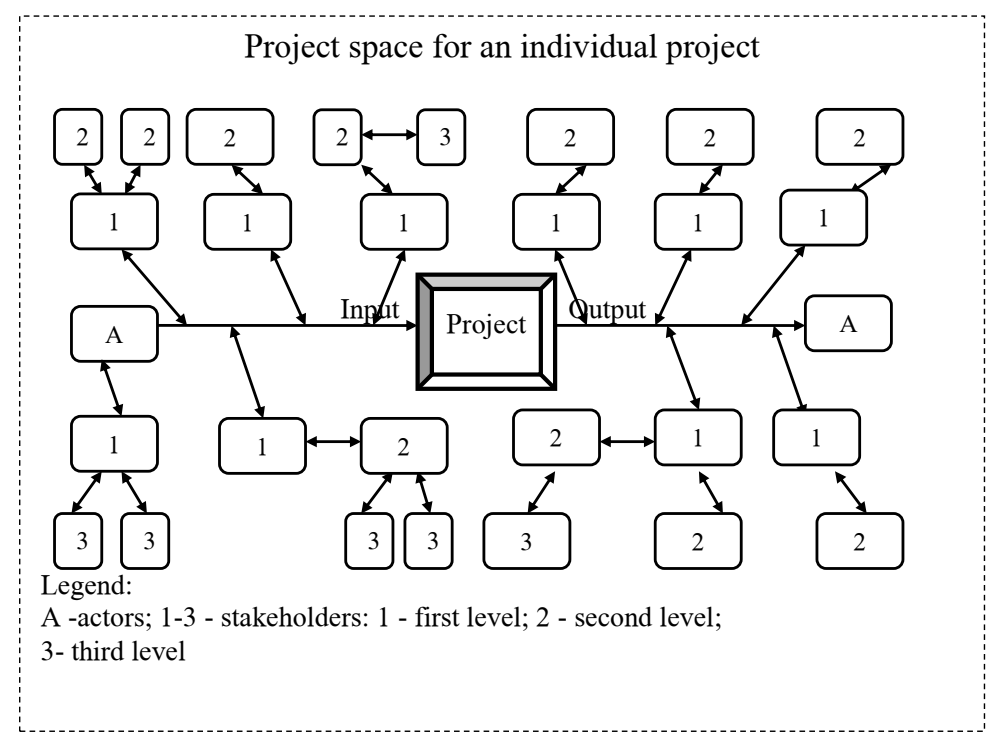

Fig. 2. Project space of an individual project

The project space of an individual project consists of the actors, stakeholders at the input and output of the project, representing: the project team; customers; consumers; investors; suppliers; the state; local community; intermediaries and other interested parties; and the connections, relationships and interactions between them.

The stakeholders at the entrance of the project are the parties interested in the supply of the products (works, services) to a new consumer in the form of a project, in the emergence of the opportunities for establishing mutually beneficial relations and prospects for their development,

Stakeholders at the output of the project are the consumers of its products, participants who create additional jobs, are the recipients of tax payments, etc.

For the purpose of analyzing the project space, it is proposed to single out the projects of different levels. The projects that provide the main result of solving the problem, which are the centers of attraction for other projects, are conventionally referred to the first level. The second level is formed by the projects that provide (or supplement) the functioning of the first level projects by means of their services (work, products), etc. (Figure 3).

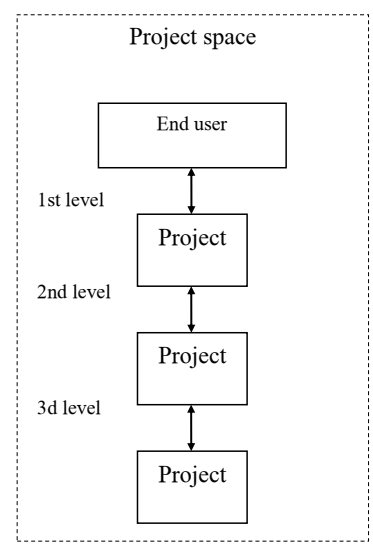

Fig. 3. Structuring the project space by the project levels 
The project space of the region can be defined as a way of manifesting the needs and interests of people, declared in a specific form of the projects on their interaction, resources, goals, rules and results.

The interaction reflects the organization of the project space, as well as the peculiarities of the processes of influence of its subjects and objects on each other, determining their mutual condition and state. It acts as an integration factor, contributing to a change in the structure and the formation of a new kind of integrity.

A fragment of the region's project space is shown in Figure 4.

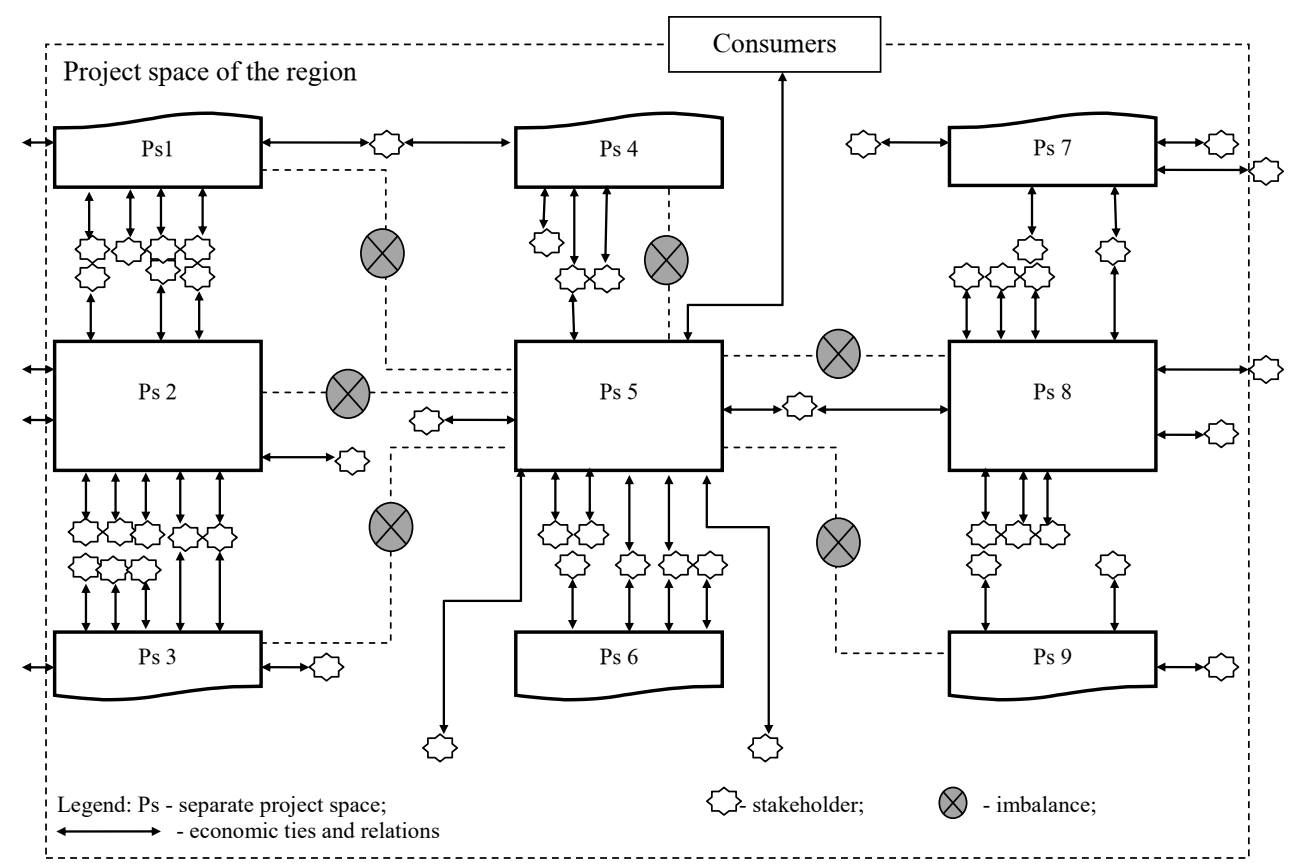

Fig. 4. Fragment of the region's project space

Resources represent opportunities (means, factors) for the implementation of targeted activities in the form of the projects.

The goals define the directions of the interactions within the framework of the project to achieve the result.

The rules establish the methods and the procedure for achieving the goal of the project.

A result is a measurable outcome, a consequence of the implementation of actions to achieve the goal of the project.

The imbalance in its generally accepted meaning is understood as the lack of balance, imbalance [30]. Integration imbalances of the project space represent a mismatch in the processes of interaction between the projects, a violation of the necessary optimality between its constituent elements that determine production and needs, the interests of various groups of the population, opportunities and norms, etc. The composition and architecture of the project space can contain a certain number of outdated, insufficiently substantiated and effective projects and other structural elements, changes in which occur in different directions, at different rates and often contradictory.

The integration imbalances in the project space are the prerequisites for reducing the effectiveness of the projects and the formation of crises in the socio-economic system of the region. 
The classification of the types of integration imbalances in the project space of the region is shown in Table 2.

Table 2. Classification of the types of integration imbalances of the region's project space

\begin{tabular}{|c|c|}
\hline Classification feature & Type of integration imbalance in the project space \\
\hline Type of project & $\begin{array}{c}\text { Imbalances of the projects: individuals and legal } \\
\text { entities; private, municipal, regional, state; monoprojects; } \\
\text { multi-projects; megaprojects, etc. }\end{array}$ \\
\hline Causes of occurrence & $\begin{array}{c}\text { Political (public); organizational; economic; } \\
\text { technological, social, etc. }\end{array}$ \\
\hline $\begin{array}{c}\text { Feature of } \\
\text { development }\end{array}$ & Stable; dynamic \\
\hline $\begin{array}{c}\text { Nature of problems in } \\
\text { the regional management } \\
\text { system }\end{array}$ & Functional; structural; system \\
\hline $\begin{array}{c}\text { Stages of the } \\
\text { reproduction process }\end{array}$ & $\begin{array}{c}\text { Imbalances of the production; distribution } \\
\text { imbalances; consumption imbalances }\end{array}$ \\
\hline Scale & General; local \\
\hline Degree & Explicit; latent \\
\hline Pattern of occurrence & Regular; irregular \\
\hline Power of influence & Light; medium; heavy (deep) \\
\hline $\begin{array}{c}\text { Duration of the } \\
\text { existence }\end{array}$ & Short-term; medium-term; long-term \\
\hline
\end{tabular}

The reasons that determine the emergence of imbalances: project space: political (mismatch of interests of the region's elite, insufficient justification of decisions, etc.); organizational - problems in the organization of project activities; economic - restrictions or irrational use of the resources in the implementation of the projects; technological unsatisfactory scientific and technological support of the projects; social - reflect the problems of social relations in the course of project activities.

According to the peculiarities of the development, an imbalance can be distinguished as: a stable imbalance, characterized by the absence of changes; dynamic imbalance characterized by the presence of continuous or discrete changes

Functional imbalances are caused by the violation of some functions and mechanisms of the regional management system. Structural imbalances are associated with the contradictions in the structure of the regional management system. Systemic imbalances are caused by the presence of the problems in the socio-economic system of the region.

The effect of the project space can be represented as a set of the effects (results) leading to a synergy - a strengthening effect of mutual influence of two or more projects:

$$
E p p\left(E_{1}+E_{2}+\ldots E_{n}\right)>\sum_{i=1}^{n} E S i,
$$

where Epp is the cumulative effect obtained in the project space; ESi is the effect obtained by the $\mathrm{i}$-th single project; $\mathrm{n}$ is the number of the projects.

Interaction of the projects will be attractive if a number of requirements are met. First, the sum of the economic benefits of an integrated project must exceed its value when compared with its stand-alone implementation:

$$
\mathrm{EPri}>\mathrm{EPa} \text {, }
$$

where EPri is the economic result of the project implementation in integrated interaction; $\mathrm{EPa}$ is the economic result of the implementation of the project in a stand-alone mode.

Second, a project space will be attractive to the regional budget if it increases the amount of tax revenues. 
To assess the effectiveness of the impact of the project space on the participants interacting during the implementation of the projects, a general approach can be proposed:

$$
E p p=\sum_{i=1}^{n} E i,
$$

where Epp is the cumulative effect obtained in the project space; Ei - the effect obtained by the i-th project due to interaction with other projects; $n$ is the number of projects interacting with other projects.

The main measures for the development of the region's project space, reducing the negative impact of integration imbalances therein.

1. Expand information support for the subjects of the project activities. An important condition for the formation of the project space is the creation of the specialized systems that constantly provide the stakeholders with relevant and publicly available information about the proposed and ongoing projects in the region. For this, websites, catalogs of projects and programs, online consulting, media reports, etc. can be used.

2. In the system of the support institutions for small and medium-sized businesses in the region, it is advisable to create a specialized organization (center for collective use, project office, etc.), providing methodological, organizational, information support for integration processes in project management.

3. It is advisable to supplement the documents on holding the competitions and providing the grants in the region, carried out by the local governments and aimed at supporting small and medium-sized businesses, with provisions on the priority of the portfolios and related projects that allow solving complex problems.

4. The strategy of socio-economic development of the region should act as an integrating basis for the use of the interrelated projects, ensuring their high efficiency and achieving synergy.

Such and other measures will make it possible to make more balanced decisions and strengthen partnerships between project management actors.

\section{Conclusion}

1. Theoretical and practical aspects of the region's project space and the manifestation of integration imbalances therein have not yet received proper actualization in the references. At the same time, the project space is a component of the economic space and the project society, the methodology of which is being developed in many countries of the world.

2. The results of the study and the elements of its novelty are: the content of the concepts of "project space of the region" and "integration imbalances of the project space" have been disclosed; a classification of the types of integration imbalances of the region's project space has been compiled; an approach to assessing the effect of the region's project space has been proposed; the main measures for the development of the project space in the region have been formulated, reducing the negative impact of integration imbalances therein.

3. Actualization of the concept of the project space in the region will increase the validity of the methodology of the project society, create favorable socio-economic consequences of the implementation of projects in the region.

\section{References}

1. E. Stillman, D. Green, Flexible project management, 464 (2019) 
2. D. Sutherland, Scrum, A Revolutionary Project Management Method, 288 (2019)

3. A guide to the project management body of knowledge (PMBOK (R) Guide - Sixth Edition, Agile Practice Guide Bundle (Pmbok Guide), Project Management Institute (2017)

4. K. Heldman, Professional project management, 728 (2012)

5. L. Davis, D. North, Institutional Change and American Economic Growth, 282 (1971)

6. M. Engwall, No project is an island: linking projects to history and context. Res. Policy, 32, 789 (2003)

7. S. Manning, Int. J. Proj. Manag., 26, 30 (2008)

8. E. Maaninen-Olsson, T. A. Müllern, Scand. J. Manag., 25, 327, Int. J. Proj. Manag., 26, $30(2008)$

9. J. Sydow, T. Braun, International Journal of Project Management, 36(1), 4 (2018)

10. K. Artto, T. Ahola, V. Vartiainen, International Journal of Project Management, 34(2), 258 (2016)

11. G. Locatelli, V. Zerjav, G. Klein, Project Management Journal, 51(5), 467 (2020)

12. J. Whyte, T. Nussbaum, Project Management Journal, 51(5), 505 (2020)

13. N. Brookes, D. Sage, A. Dainty, G. Locatelli, J. Whyte, International Journal of Project Management, 35(7), 1213 (2017)

14. T. Dille, J. Söderlund, S. Clegg, International Journal of Project Management, 36(5), 673 (2018)

15. B. Flyvbjerg, Project Management Journal, 45(2), 6 (2014)

16. G. Locatelli, M. Mikic, M. Kovacevic, N. Brookes, N. Ivanisevic, Project Management Journal, 48(5), 78 (2017)

17. S. Cicmil, Proj. Manag. J., 37, 27 (2006)

18. L. van den Ende A. van Marrewijk. Int. J. Proj. Manag, 02.007 (2004)

19. S. Lenfle, J. Söderlund, Organization Studies, 40(11), 1713 (2019)

20. W. R. Scott, R. E. Levit, R. J. Orr, Global projects: Institutional and political challenges (2011)

21. R. A. Lundin, Project Management Journal, 47(4), 7 (2016)

22. G. Winch, R. Leiringer, International Journal of Project Management, 34(2), 271 (2016)

23. D. Sage, A. Dainty, N. Brookes, D. Andrew, B. A. Naomi, International Journal of Project Management, 32(4), 544 (2014)

24. A. Davies, T. Brady, International Journal of Project Management, 34(2), 314 (2016)

25. P. E. Eriksson, M. Westerberg, International Journal of Project Management, 29(2), $197(2011)$

26. D.S. Abd Razak, G. Mills, A. Roberts, Project Management Journal, 51(5), 474 (2020)

27. Investment projects. JSC "Corporation for investment development RSO-Alania". http://www.alania-invest.ru/projects

28. Innovative projects. JSC "Corporation for investment development RSO-Alania". http://www.alania-invest.ru/page/innovacionnye_proekty

29. Z.L. Dzakoev, V.T. Balikoev, Catalog of innovative and investment projects of the Republic of North Ossetia-Alania (1997)

30. Cambridge Dictionary, https://dictionary.cambridge.org/ 